\title{
Spaces for Participatory Design Innovation
}

\author{
Gemma Teal \\ The Innovation School, The Glasgow School of Art, \\ Glasgow, Scotland \\ g.teal@gsa.ac.uk
}

\begin{abstract}
This paper presents a model to guide the design of participatory design (PD) projects, which was generated through methodological reflection on a programme of digital health and care research. Building on wide use of the term spaces within PD literature and theory, the model comprises seven spaces that can be designed to support diverse stakeholders to engage in a PD process. The model encourages reflection on the capacity of participants to critically and creatively engage with the concepts being proposed, in order to design a process to scaffold participation. Aiming to support PD practitioners and researchers to identify the combination and sequence of hybrid spaces required to move participants and concepts towards resolved designs, the model guides the careful design of each space supported by examples of practice. The model is also designed to enable reflective practice and articulation of $\mathrm{PD}$ processes to support integration within multidisciplinary collaborations.
\end{abstract}

\section{CCS CONCEPTS}

- Human-centered computing $\rightarrow$ Human computer interaction (HCI) ; HCI design and evaluation methods ; User models; Interaction design ; Interaction design process and methods ; Participatory design; Interaction design ; Interaction design process and methods ; Contextual design.

\section{KEYWORDS}

Participatory Design, models, methods, design space

\section{ACM Reference Format:}

Gemma Teal and Dr. Tara French. 2020. Spaces for Participatory Design Innovation. In Proceedings of the 16th Participatory Design Conference 2020 Participation(s) Otherwise - Vol 1 (PDC '20: Vol. 1), fune 15-20, 2020, Manizales, Colombia. ACM, New York, NY, USA, 11 pages. https://doi.org/10.1145/ 3385010.3385026

\section{INTRODUCTION}

Terms such as collaboration, co-production, and co-design are becoming heavily featured in the landscape of policy, practice and service innovation. Engaging citizens in the design and development of policy and public services is increasingly advocated to

Permission to make digital or hard copies of all or part of this work for personal or classroom use is granted without fee provided that copies are not made or distributed for profit or commercial advantage and that copies bear this notice and the full citation on the first page. Copyrights for components of this work owned by others than the author(s) must be honored. Abstracting with credit is permitted. To copy otherwise, or republish, to post on servers or to redistribute to lists, requires prior specific permission and/or a fee. Request permissions from permissions@acm.org.

PDC '20: Vol. 1, fune 15-20, 2020, Manizales, Colombia

(c) 2020 Copyright held by the owner/author(s). Publication rights licensed to ACM

ACM ISBN 978-1-4503-7700-3/20/06 ..\$15.00

https://doi.org/10.1145/3385010.3385026

\author{
Dr. Tara French \\ The Innovation School, The Glasgow School of Art, Forres, \\ Scotland \\ t.french@gsa.ac.uk
}

empower citizens and respond appropriately to social and political challenges [20]. Despite this, the balance of power remains top-heavy.

In the health and care landscape, a similar picture is emerging with increasing use of design-based approaches such as ExperienceBased Co-Design (EBCD) [2, 10, 25], design thinking [14] and other design-centred approaches $[17,42,83]$ that seek to involve, in some way, patients and staff in a design process. While the use of these approaches is widespread and growing, a worldwide study of their implementation found that the application of co-design activities varied widely, in some cases with limited active participation from patients [25]. Moreover, there is growing concern that despite increasing awareness, there has been a dilution of the meaning of participation [73]. The complex nature of the social challenges being tackled require multidisciplinary collaboration [60] therefore being able to describe and integrate methods within a clear process, and a shared understanding of language for participation are needed. While toolkits and step-by-step processes can be useful, they must be accompanied by a 'participatory mindset' [68] and honed skills to identify where people can contribute, and adapt methods and tools to the specific context [56]. Although many examples of design-based approaches and methodologies exist, there remains a lack of guidance to support the way in which participation at a project level can be designed, and to encourage reflection to achieve the full potential of user involvement in the process [8]. There are however many useful publications offering guiding principles for participation (e.g. $[13,56])$, but these do not offer a flexible structure for considering how to apply the principles in practice. Organising participation is challenging, requiring communication, negotiation and compromises, therefore design researchers (DR) need models which can support this [11], particularly in contexts of increasing complexity.

Within the wider field of public health, the emerging need to translate research findings into tangible, evidence-based interventions [41], and the lack of methodological guidance on how to integrate evidence systematically from various sources remains a challenge for the research community [61]. Participatory and co-design approaches offer a means of mobilising and creatively engaging individuals and communities towards change, however there is no explicit and accepted description of their application [61], nor is there an understanding that the evidence they provide is sufficient to impact policy and practice. As a result, knowledge of how to integrate these approaches within existing research design is required, with potential to achieve high impact across design and the social sciences.

The aim of this paper is to introduce a model to support DRs and multidisciplinary research teams to collaborate in the design of participatory innovation processes. We provide an overview and critique of literature documenting frameworks, models and 
lenses for PD, share the methodological reflection that led to the development of the model and present the model, illustrated with project examples and discussed in relation to existing literature.

The model proposed in this paper has been developed within the context of health and care innovation. We are keen to engage the PD community to understand the contribution and value of this model for design education and the wider field.

\section{FRAMEWORKS, MODELS AND LENSES FOR PD}

There have been a number of efforts to articulate organising principles for PD. These are generally offered with the acknowledgement that they are not intended as 'recipes' to be followed to the letter, but "general guidelines that must be carefully selected, adapted and appropriated to the specific project and situation at hand" [13]. While not claiming to be comprehensive, we have searched databases for papers that relate specifically to participatory and co-design, using the terms: framework, model and lenses. We ruled out highly specialised frameworks which were not considered to be widely applicable.

\section{Linear frameworks and models}

Bratteteig et al. [13] provide a helpful overview of organising principles and guidelines for PD, which have emerged from HCI and software development. The MUST method was developed for IT development in an organisational context [52] and offers a conceptual framework of the design process. It addresses aspects of project management and communication, and advocates a five-stage linear process. Drawing on ethnographic methods, it seeks to gain an understanding of the current, and generate a vision of the future to guide development. While the principles of this approach are insightful, and the stages applicable in PD more broadly, the method is specifically tailored to IT development. In addition, the framework works towards achieving the desired outcome, rather than achieving meaningful participation of diverse groups. The Software Technology for Evolutionary Participatory Systems Development model [29] is a "process-oriented paradigm", designed around the software development cycle. The model is very prescriptive, and describes where participation should take place in the cycle, rather than how it should be designed.

Contextmapping [80] explains how to conduct PD projects, prescribing sequential stages of: preparation, sensitisation, group sessions, analysis and communication, alongside highly practical guidance on how to design each stage. Advice offered ranges from how many participants to include in a session, how to record and analyse the data, and how to communicate the outputs. Although useful and accessible, it focuses on advice about the design and facilitation of a specific sequence of activities, when in practice PD can take many forms.

Within the context of health and social care, $\operatorname{EBCD}[2,25]$ is a PD model mainly focused on service improvement rather than innovation. The six stage process includes: i) project set-up, ii) observation and in-depth interviews with staff, iii) filmed narrativebased interviews with patients and carers edited into a 30 minute film, iv) staff, patients and carers identify priorities for improvement 'triggered' by the narrative film, v) groups of patients and staff work on the identified priorities, and finally vi) a celebration and review event. This approach has been widely implemented and adapted [55], but the levels of participation in design have been found to vary widely [25]. Along similar lines, Akoglu and Dankl [1] proposed a framework to enable participation and collaboration among doctors, nurses, patients and relatives. This framework outlines four phases (MOVE: Meeting stakeholders, switching Over roles, Voice ideas and Evaluate) which provide: i) spaces to enable empathy amongst diverse stakeholders; ii) a workshop design which enables common ground; and iii) thoughts on the changing role for the DR to that of mediator.

These frameworks offer linear scaffolds for PD, albeit admitting that in practice activities may overlap. While undoubtedly easier to follow and communicate to non-designers, this over simplifies the process. There is a need for a more flexible model that leaves space for creativity in adapting methods and tools to suit project-specific contexts.

\section{Non-linear frameworks and models}

Sanders, Brandt and Binder [69] offer a framework that provides practical guidance about how to decide which methods, techniques and tools to use when designing a PD session. The framework advises DRs to first consider the specific purpose of participation and groups methods and tools in terms of the form that participation takes (making, telling and enacting). It offers modes of participation appropriate to individuals and groups and is inclusive, in that it is open and can be added to as new methods and tools are developed. The framework is highly flexible, allowing for the combination of different methods and tools. By conceiving of the entire experience, the framework supports DRs to consider holistically what the participants will encounter, seeing it as a sequence of activities each building on the last to enable meaningful participation. This is a practical and useful resource for PD, focusing on the activities of collaborative design. It does not however address some of the more experiential and relational aspects of participation, or how to create the conditions for these activities to take place.

Referring back to Bratteteig et al.'s discussion of PD organising principles for software development, there are two non-linear models [13]. Cooperative Experimental System Development [44] offers a conceptual model which is visualised as layers, mapping project activities (top layer) onto the project concerns (middle) all driven by the overarching goal of the project (bottom). This meta-method is useful in aligning participation to project goals, and focuses on the transformation of loosely specified prototypes into engineered computer systems. Although the model was designed for system development, the concerns and activities are broad enough to be transferable. However, the model lacks guidance on how to design participation, being more focused on what and why activities are required. Use-orientated design [24] offers an iterative cycle for PD which places importance on the DR working with users to understand current and future use. Through iterative prototyping and testing, the cycle results in a clearly defined set of requirements. This model outlines a process for active participation in development, however there is scope to give the DR more flexibility, allowing for other forms of participation.

Working in the context of PD with young people (YP), Druin [27] developed a model of participation which considers the role of participants as user, tester, informant or design partner. The 
framework intends to support DRs to make informed decisions about the process for PD with YP. It was further developed to consider if participants are included, consulted or empowered [48]. Although this is centred on PD with YP and intended for evaluation rather than scaffolding $\mathrm{PD}$, these classifications could be broadly applicable and useful in setting goals for participation.

These frameworks and models reflect the non-linear nature of PD projects and attempt to guide participation by representing it as iterative cycles [24], focusing on the purpose of activities and linking to project goals $[44,69]$, and defining different modes $[27,48]$ that can build to enable meaningful participation [69].

\section{Lenses to guide PD}

Brandt [11] proposes design games as a framework for organising participation in PD projects. Building on the Future Workshop [51], the games are designed to enable critique of the current, envisioning of the future, explore implementation, and provide a basis for mutual learning. The framework also proposes "certain ingredients and frames in relation to how to stage participation". While this framework provides a wealth of flexible ideas for designing participation through the lens of games, this is only one of many possible approaches to engagement. For sensitive contexts (e.g. working with people who have experienced miscarriage), conceiving of participation as game play may not be appropriate. In addition, while many stakeholders may be involved in PD, they may not be involved at the same time - the dialogue may be a one-to-one interaction between the DR and the user and therefore a game style approach would not be the most conducive.

Chin et al. [19] use scenarios as a 'task-artifact' framework for PD in HCI development, inviting participants to analyse their own usage scenarios to determine requirements and envision features and future use. By facilitating participation through scenario-based design, the framework acknowledges users as the expert: the scenarios exemplify their practices and requirements. However, this approach relies on a clear definition of the current or desired usage scenario for $\mathrm{HCI}$ design, which is rarely the starting point for $\mathrm{PD}$ in complex social contexts.

Frauenberger, Good and Keay-Bright [30] suggest phenomenology as a framework for PD, placing the focus on understanding experience in order to design interaction. This approach frames $\mathrm{PD}$ as an "interpretive and generative process" and offers a way of thinking holistically and critically about design practices. The focus moves away from the specification of design outcomes to "create interactive environments that scaffold the process" of participants contributing to the design. It advocates the use of figurative or metaphorical concepts to enable participants to inform design without the need to understand technical concepts. This contribution is exciting because it moves beyond the literal design outcomes and engages with the participants' lived experiences to inform design. This is particularly suited to contexts where participants may not have the functional ability to express their ideas, and where the concepts being developed are difficult to communicate. However, while it offers a useful overarching approach to think creatively about how to engage, there is a need for guidance on how to implement this in practice.

\section{Lenses and frameworks for critical reflection}

In subsequent work, Frauenberger et al. [31] developed a tool for critical reflection on PD projects comprising four lenses: epistemology, values, stakeholders and outcomes, and examining coherence between the lenses. The tool aims to ensure quality and build arguments for the value of PD in terms of accountability and rigour. It is intended for use in planning, and for reflection during and post. Although the lenses are clearly relevant to participation, the tool does not offer any guidance on how to design for participation. In a similar vein, Kyng [53] introduces a conceptual framework which aims to "bridge the gap between politics and techniques" within PD for ICT design. In order to increase the influence of PD approaches in this context, the framework promotes discussion and relation of nine elements: ideals, company roles, intellectual property rights, funding, users, settings, safeguarding user interests, project outcome and finally techniques. Again, this is not attempting to scaffold participation, and appears to be intended for securing funding, anticipating political challenges and for reflection.

Saad-Sulonen et al. [65] offer temporal lenses to critically examine participation, as an alternative to the activity based frameworks and models presented here. This was a reaction to the sustained duration of some PD initiatives over many years and the possibilities this enables in implementation and evaluation, and PD in use. They propose five lenses that support DRs in considering the temporal dimensions of participation: the phasic, emergent, retrospective, prospective and long-term lenses. They advise ways these lenses can be used to consider participation at different stages of the project (loosely before, during and after). It is not intended as a tool for designing or organising participation, but for reflecting on and understanding how participation unfolds over time.

Drain, Shekar and Grigg [26] have developed a framework for use in the evaluation of PD projects. The Insights, Solutions and Empowerment framework was developed for the evaluation of PD for humanitarian technology development. In the context of the project's values and ideology, the framework considers the knowledge produced for design (insights), the designed outcomes (solutions) and the empowerment of participants. This offers a useful structure for evaluating participation in completed projects, but does not attempt to guide the design of participation. Similarly, the PartE Framework [39] is a tool purely for rigorous evaluation within and across projects, which considers: objective, practice, interaction, barriers, representation and impact.

These lenses offer perspectives on creating the conditions for participation at a project level $[31,53]$ and/or act as tools to retrospectively evaluate the impacts and quality of participation $[26,31,39,53,65]$. They are open enough to apply to different types of projects, but offer limited guidance on how to structure and design participation.

\section{Opportunities for further guidance}

PD practice relies on a participatory mindset [68] which guides the DR in carefully appropriating and combining methods in a creative response to the context. This requires a level of skill and experience, and a holistic view of how each encounter builds to create meaningful participation towards the project aims. There is a need for guidance to support DRs to conceptualise and articulate PD 
practice. While the frameworks, models and lenses explored here offer useful ways of structuring, reflecting on and evaluating PD, there is a need for a more open and flexible model which scaffolds the design of participation across a project, and enables reflection and clear articulation of this complex process.

\section{DEVELOPMENT OF THE SPACES MODEL}

The 'Spaces for PD Innovation' model (see Figure 1), herein referred to as the Spaces model, embodies the methodological reflections of our transdisciplinary team, spanning design, social science and computer science disciplines. The model emerged from reflecting on a design research programme led by The Glasgow School of Art as part of the Digital Health and Care Institute, an innovation centre in Scotland. The programme aimed to support innovation in health and care through PD by involving all stakeholders and users in the design of products, services and systems to ensure they are driven by user needs [35]. Over five years, the team delivered in excess of 25 projects across a breadth of challenges in health and social care [21]. Projects aimed to use a collective understanding of the current experience to consider preferable new ways of working and caring, enabled by technology [36]. They also engaged around speculative technologies, using design-led approaches to translate technical innovation into meaningful experiences to enable participants to consider how they might impact on e.g. the management of their long-term condition, support more meaningful interactions with services, or change working practices [76]. This has enabled the team to develop context specific knowledge about crosscutting challenges and opportunities in this landscape. Alongside this, the team gained a wealth of experience of how to engage diverse participants in PD. The Spaces model articulates how we create the 'right' conditions to meaningfully involve participants in health and care innovation.

The use of the Spaces model as a tool for reflection was explored with a group of DRs during an internal team workshop focused on understanding ethical practice. The Spaces were made tangible on Perspex discs so that DRs could capture key elements of each Space by reflecting on individual projects, and use the discs to build physical representations of the values, processes, methods and approach to participation. The reflections captured during this workshop provided validation of the nature of the model and further defined the spaces and core values.

The model was also introduced to students as a structure to understand when and where to use different methods and approaches, alongside a set of questions to consider pre- and post $\mathrm{PD}$ project. The exploratory session invited students from several design schools to use the model to consider how to apply PD within their current project. Informal feedback from the students suggested that the model offered a welcome structure to support them to consider how to choose from the many different methods and approaches they were exposed to in their training.

\section{WHY HAVE WE USED THE TERM SPACES?}

Muller [60] introduces PD as the "third space" for users and technologists to share knowledge of their own distinct spaces (i.e. software development and the users' work domain) and generate new ideas. This "hybrid" third space is a fertile environment for mutual learning, challenging assumptions and discussing differences. Muller
[60] surveyed the methods, tools and approaches used within PD and described the attributes of the third space they aimed to create. Björgvinsson, Ehn and Hillgren [4] propose that the role of PD is to create "infrastructuring agonistic public spaces" that hold diverse stakeholders in "mutually vigorous but tolerant disputes". This perspective on PD emerged in response to the challenges of applying it in new contexts, beyond workplace projects where this field developed, to innovation research within the public realm. By focusing on the infrastructure or spaces that need to be created for PD within more diverse groups of stakeholders, the authors democratise innovation for the public sphere and broaden the scope of their PD practice from technical innovation to social innovation. For Sevaldson [72], the term "Design Space" refers to the setting for research, which includes the physical, spatial, technological, cultural, and social conditions for the design process. Within the field of HCI, spatial perspectives on design and interaction have enabled the development of concepts such as "multidimensional design spaces" that empower users to engage in the design process and continue to evolve products in use [40]. Benyon [3] introduces "Spaces of Interaction, Places for Experience" to consider the space within which users interact with technology, "placing people in the context of a technological world" and understanding how this affects the user experience.

Groves and Marlow [45] offer "Spaces for Innovation" as the physical, digital and social contexts which support innovation within an organisation. This model aims to support organisations to embed conditions for innovation that suit their specific goals. It is not a model for PD, but focuses on building cultures of innovation within organisations. While this context is different from our own, the term spaces is used to support leaders to see their role as creating the conditions for creative collaborations, which has parallels to what we are trying to achieve in PD.

Many PD researchers highlight the need for "safe spaces" [16], in particular within vulnerable or underserved groups [46], sensitive topics and where power between participants and/or DRs is imbalanced. Akoglu and Dankl [1] describe the role of the DR in providing egalitarian "participatory spaces" to enable empathy building and mutual learning.

Therefore taking a spatial perspective on PD and innovation is not new. We argue that this is a reaction to the complexity of the concepts being discussed: through reification of intangible processes and conditions for PD as 'space', we make it easier to understand. We give the conditions for participation a tangible form, as the output of the DR's efforts. Beyond the need to create safe, egalitarian spaces where participants feel comfortable and able to share their experiences and ideas, there is a need to consider how the choices made can influence the type of engagement and insight that can be achieved. This starting point allowed us to reflect on and communicate how our programme approached the design of projects, and the types of conditions that supported us to achieve meaningful participation with innovative results.

\section{WHAT ARE THE SPACES?}

The Spaces model comprises seven spaces that can be designed to support a range of diverse stakeholders including end users to engage in a PD process. Figure 1 offers a visual representation of 
the Spaces model, intended to communicate how the seven spaces can be layered and combined, with consideration of the physical setting to support each space and underpinned by a cross-cutting set of values. The model is non-linear: it is designed to support projects to move through the spaces in a bespoke order, skipping or revisiting spaces to suit the context, participants and concept being explored. The ordering of the spaces within the visual is not intended to suggest a sequence, and is presented in a horizontal orientation to indicate how different spaces can build on each other to scaffold higher levels of critical thinking amongst participants.

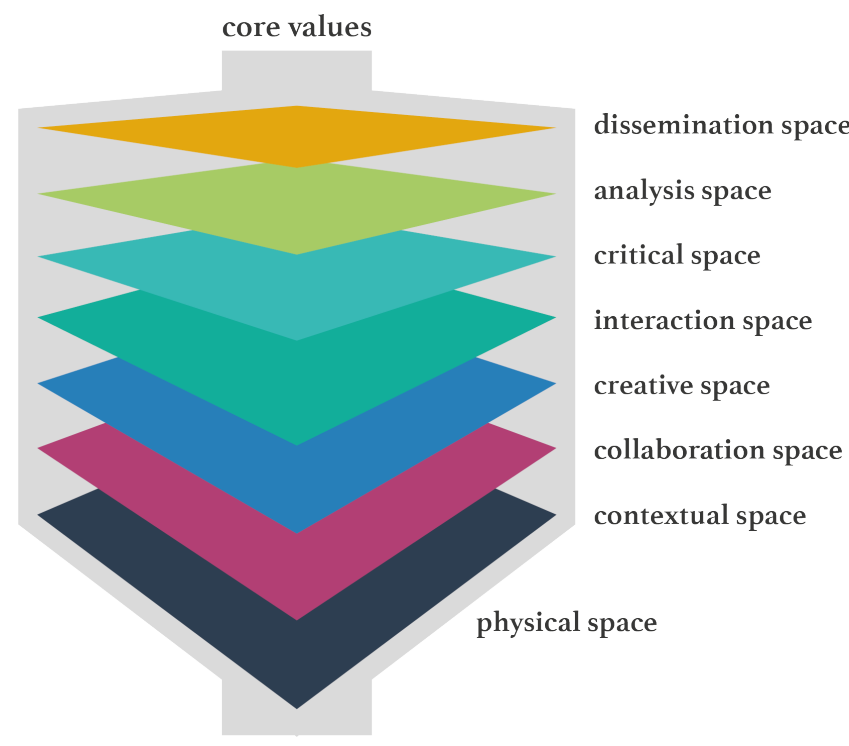

Figure 1: Spaces for Participatory Design Innovation

As with the many other PD frameworks discussed, our model is accompanied by a set of core values. These build on the guiding principles of $\mathrm{PD}$, but have been augmented based on reflection on the values of the transdisciplinary team, and what is distinct about our approach. The following section will provide a discussion and critical reflection of each space, beginning with an overview of the qualities and values of the model.

\section{The Core Values and Qualities of Design}

Across all spaces, we have qualities and values that guide our ways of working internally and externally to ensure an ethical practice, i.e. empathy, equality, building trust, safe spaces and creating comfort in uncertainty. Another important quality relates to aesthetics, where we aim to attune the aesthetic of our materials to the specific context. This is a sensibility of our work that aims to support interaction with and communication of the subject matter we are exploring. It is also of great importance when inviting participants to share their lived experiences. Attention to aesthetic means being sensitive to the subject matter by conveying the right emotional tone through tools designed to support dialogue. For example, in a project which explored women's experiences of miscarriage, an aesthetic for engagement materials and tools was designed using warm colours, soft, tactile materials and shapes. Using the metaphor of a dream-catcher, participants were invited to choose from carefully selected symbols to attach to their dream-catcher to represent and discuss their own experience [63]. In many other projects we use a sketchy and unfinished aesthetic, deliberately different from the clean icons and colour palettes used in digital health products. This is intended to convey to participants the exploratory nature of the projects, ensuring materials are open to be shaped through participation, and challenging perceptions that digital health products need to follow existing design conventions.

The ability to improvise and adapt our practice 'in the moment' is a key quality developed through the experiential nature of our work. This has been cultivated through the relationships amongst the team, where we have built an understanding of our individual strengths and skills, and how we adapt and apply these as a team within projects.

\section{The Physical Space}

This is the foundation for creating all other spaces. Considerations include using real or realistic environments to enable the development or testing of concepts in use, and creating conditions conducive to creativity and collaboration using informal, neutral or inspiring physical spaces. The physical space is also considered whilst being mindful of our engagement and participation intention. Often we hold activities in spaces that are local or familiar to participants to support them to be able to engage and participate. As Muller [60] describes, "one of the simplest parameters that can be manipulated to influence hybridity is the site of the work". The physical space can be used to create collaboration amongst a diverse group, by disrupting existing power dynamics and taking people out of their normal environment e.g. bringing together health professionals and patients outside of the clinical setting.

Careful consideration goes into not only the choice of the physical space for engagement, but also the design of the environment. Decisions on where to hold the sessions are made in collaboration with project partners who often support recruitment. Given they may already have an existing relationship with participants, they can often recommend places which are familiar or convenient for participants. For example, in a project exploring co-design of a game-based learning tool with YP with learning disabilities [78], project partners who supported the YP recommended a local venue which was known to participants. This supported the YP to feel safe and made it convenient for them to travel to a familiar location.

The design of the physical space is important to support participants to feel able to engage. This can involve creating a space that is conducive to the type of activity being employed. For example, when undertaking interviews or one-to-one engagements, it is often appropriate to visit participants in their own space or at a familiar venue. In terms of group and creative activities, there is a need to create an inspiring space which can involve preparing design materials that are stimulating to participants when engaging in co-design. Finally, when engaging participants in activities that aim to ideate, prototype and test, it may be necessary to create a physical representation of a space. For example, in a project exploring the concept of a sensor-based notification system with older adults [33] it was firstly important to engage participants individually in their own homes to understand everyday life and use of technologies. To support participants to think about what 
was important to them in terms of products and allow them to engage in available everyday technologies, we organised a guided tour of the home and technology sections of a department store. Finally, to test the emerging concept, a mock home environment was created to allow participants to interact with the system and provide feedback and ideas.

\section{The Contextual Space}

The contextual space involves understanding end users, their environments, cultures and lived experience. This might be achieved by employing ethnographic methods such as observations, situated interviews and cultural probes [38]. Building a contextual understanding can also be achieved through mapping activities or role-play. Many of the methods presented are used in traditional research, but in a PD context there is more emphasis on how the method is applied as part of a wider process. In parallel to participatory methods, literature review and horizon scanning also develops a wider contextual awareness.

The contextual space is a vital part of knowing how to open up the design process to a diverse range of participants and can assist in ensuring any subsequent spaces are built on the core qualities. Existing approaches such as 'Contextual Design', which seeks to generate rich insight by understanding users and their environments [47] are more similar to user-centred design approaches with a lack of direct participation from users in the process. In the Spaces model, immersion is achieved by embedding in the context and involving people in building this understanding, often using multiple methods to ensure a rich, in-depth picture.

The activities within the contextual space are dependent on the work that has gone before, e.g. evidence from previous research or the experience of partners in proposing the innovation. As such, the purpose of the contextual space is not to answer the research question, but to ground it based on a rapid, targeted, deep dive into the subject matter. By gaining an understanding of the here and now, the contextual space can support innovation through imagining "what could be based on the knowledge of what is" (Work Practice and Technology group a Xerox PARC, in [6]).

The contextual space could be described in terms of "critiquing the present" [50], however in the Spaces model this is not necessarily the starting point for projects. Learning about the present can sometimes be best achieved through intervention [52], therefore the contextual space can be designed to follow critical or interaction spaces to enable participants to engage with what aspects of the current are relevant to the proposed innovation.

Illustration of the Contextual Space: In a project which aimed to develop a digital directory of services with ambulance clinicians [32], DRs used a number of activities to gain a contextual understanding of current practices. The DRs engaged in design ethnography, shadowing clinicians to understand the technologies and processes involved when responding to an emergency. This provided insights to inform the design of subsequent spaces, specifically in understanding the participants' descriptions of the current challenge and ideas.

\section{The Collaboration Space}

For PD in health and care contexts with diverse stakeholders, participants are required to engage both with the concepts being explored, and with each other's points of view [34]. This space is focused on building relationships and addressing any power dynamics that could potentially arise by bringing specific groups of participants together. Empathy plays a crucial role within this space: DRs need to be able to empathise with the participants, and the participants with each other [34]. An overriding concern of the collaboration space should be to create a feeling of safety, supporting the sharing of experiences, enabling the individual voice to be heard while working towards a collective understanding [77]. Inclusivity is a key concern, ensuring diverse groups of participants are supported to make equal contributions. Asset-based approaches and experienced facilitators empower participants to contribute their individual capabilities [22], opinions and ideas [75]. Ludic design approaches [37] and bespoke games [11] are also highly effective. Experienced facilitation is required in surfacing and managing possible tensions caused by differing perspectives, and turning this into productive, agonistic dialogue that allows differences to be raised [4].

Illustration of the Collaboration Space: For a project involving YP with learning disabilities in co-designing a game-based learning tool [78], it was important to build trust, create a safe space and ensure participants had the opportunity to have their ideas and experiences heard. This involved the DRs building a relationship with participants to support the collaboration over the course of the project. To do this, the DRs spent time with the project partners to understand the current learning programme and prepare the initial engagement materials to ensure appropriate language and terminology. To introduce the project to participants and share information to invite participation, the DRs joined a social event regularly attended by the YP. The DRs then organised another event for YP to gain more information and ask questions before signing up. This allowed the participants to get to know the DRs and begin to build trust. Building this relationship also supported the design of tools and activities.

\section{The Creative Space}

The creative space involves generative activities [68] that are intended to engage participants directly in the design process. This often includes the use of generative tools [66], materials for making and prototyping with participants [23] and methods such as bodystorming [62] i.e. designing during role-play in realistic settings. The activities are designed to tap into latent design skills and support participants to imagine possibilities by working through ideas. They encourage thinking through making, with design tools and materials often taking on a sketchy, unfinished aesthetic to invite participants to add their own ideas. In this space the physical environment for co-design activities is of particular importance. This can be as simple as having a range of craft materials, objects and play materials available to participants to inspire and support them in creativity. Scenario-based design [18] derived from stories and experiences captured in the contextual space is often used to inspire thinking about what could be different. Design toolkits [54] and exemplars from parallel contexts can be used to inspire participants. 
Design facilitation within the creative space aims to provide guidance and support to participants when engaging in co-design. It is also where the DR can bring their skills as a designer, actively listening and responding by proposing ideas to be discounted or developed by participants. They also play a role in giving form to participants' ideas, sketching, and prototyping with participants as a form of experiential practice [81]. Facilitation can be in small groups (as per Visser et al. [80], we find four to six participants per group is optimum), one-to-one facilitation or self-facilitation through the use of a design canvas [49].

Illustration of the Creative Space: Within a project that sought to understand how people living with a long-term condition would like to manage their data [76], we developed an activity using a bespoke tool to manifest their ideas. Participants were supported by a DR on a one-to-one basis. Beginning with a card sorting exercise, the participants grouped data and put it into cardboard pockets, which were then connected up to show hierarchy and data sharing. Finally, the pockets were placed in a 'Backpack' which the participants personalised, adding security and care instructions. The tool was assembled by the DR, instructed by the participant, which enabled them to focus on their ideas. The resulting artefact resembled a wireframe of a personal data store, which was later translated into a digital prototype for subsequent testing.

\section{The Interaction Space}

This space aims to model and understand interactions between people, products and systems employing methods such as 'wizard of oz' and experience prototyping [15], and observing how participants respond and change their behaviour [5]. Close to what Muller [60] describes as "constructions", this also bleeds into the critical space in that it provides an experience to reflect on and give critical feedback. This may be considered the least participatory of the spaces, as participants are observed rather than directly engaged. This is mediated by how the activity is introduced, with the clear aim of inviting their critique as experts in the context. Although the settings are as realistic as possible, we often retain a sketchy, low-fidelity aesthetic to physical products and interactions, to invite open critique without the participants feeling the design is resolved. Prototypes can be iteratively refined and tested during a session, enabling participants to see and test their input in the maturing products and interactions [68].

Illustration of the Interaction Space: During the project that sought to explore a sensor-based notification system for older people, we created a wizard of oz prototype within a realistic home environment [33]. The project followed contextual spaces within home and department store environments (as previously described), and therefore the prototype embodied interactions, aesthetics and technologies inspired by earlier phases of the project. The prototype also incorporated personalised elements from each participant's home (such as accurate scenarios, familiar voices) to provide as realistic a representation of use as possible. The DRs were able to observe the interaction, and record for later analysis.

\section{The Critical Space}

Reaching the critical space often requires participants and DRs to move through contextual, collaboration and crucially interaction spaces in order to scaffold participants to enable them to meaningfully engage with new concepts and critically reflect based on their lived experience. Earlier spaces may be "necessary for users to be able to form and voice their opinions" [13], and enable decisions to be grounded in use. For DRs, reaching the critical space may require earlier spaces to understand and translate critique into design decisions. This process is described in terms of "mutual learning", where the DRs share their knowledge of design and technical issues and participants share their knowledge of the use context [13].

PD has a wealth of approaches to support critical thinking amongst participants. Through Reflective Design, "unconscious aspects of experience" are brought "to conscious awareness. . . thereby making them available for conscious choice" in the PD and ongoing use of technology [71]. This can be achieved by creating a space to reflect on the experience of using new technologies (i.e. in the interaction space) or through approaches such as Critical Design [28] which use artefacts [9] designed to stimulate critique and offer a different perspective. Stories can encourage reflection on current and future worlds, which may be told through the medium of storyboards, vignettes or pre-made films. Design fiction [7] can be created to bring concepts to life without the need to prototype, showing fictitious and often provocative or playful new products or services in use and exploring the reaction of the audience. Lowfidelity or deliberately ambiguous prototypes invite participants to be more critical than highly polished prototypes [64], where meaning is "revealed through the stories told about it and the scenes in which it plays a role" [68]. Where proposed new concepts may be highly technical, metaphors [30] can be used to relate the intended functionality to familiar products. This removes the need for participants to relate to technical language.

The critical space can also be designed to encourage reflection on current ways of working and living to identify opportunities for innovation. Visual representations can be created (e.g. through collaborative mapping) to enable the participant to 'see' and reflect on the whole system which they may only currently see a small part of [57]. Findings may be shared from previous spaces which offer external insight, encouraging discussion and reflection on meaning and values. This can include methods such as 'trigger films' used in EBCD, whereby compilation films of patients describing their experience are used to support health professionals to reflect on the care they deliver, and build empathy prior to collaborative working $[2,25]$.

Illustration of the Critical Space: In a project which explored the design of a music-based tool to support communication and relationship building among YP with Additional Support Needs and their families and carers, a series of speculative prototypes were developed that embodied the knowledge generated through the contextual space. These artefacts aimed to communicate the concept of a music-based tool and support the YP, families and carers to critically evaluate the concept and test whether these artefacts supported the project objectives. While the prototypes were not intended as finished products, they enabled participants to engage with possible interactions which were developed in response to the 
behaviours observed and the opportunities identified. They communicated the overall concept in a tangible way, making it more accessible to the participants. Here the critical space enabled the carers and family members to reflect that while there were many possible manifestations of the music-based tool, what they really needed were accompanying resources to give them confidence in using the tool to create meaningful interactions through music.

\section{The Analysis Space}

This space involves analysing the data collected to inform development by translating insights into design implications, outcomes and requirements. It aims to ensure that the appropriate analytical techniques are used with respect to the type of data collected (which can include e.g. transcribed audio recordings, annotated design tools, video footage and prototypes) but also in terms of how findings will be translated into insights, actions and tangible artefacts. At the same time, it seeks to balance this with the need for more rapid techniques, particularly during iterative cycles of analysis in between engagements. The analysis space is where consideration is given to how knowledge may be embodied through other forms to support communication and roadmapping towards change.

Activities should seek to open up the analysis space and meaning making to the participants, through activities which support "cointerpretation" [47]. In order to achieve this, we use visual means of recording live dialogue such as graphic facilitation [79], concept mapping [82], rich picture [59] and bespoke design tools [35]. This enables the DR to share their interpretations and let participants respond, "tuning and correcting understanding along the way" [47]. Translating findings into prototypes turns them into "tools for analysis" [13], used to validate understanding.

Illustration of the Analysis Space: Within a project that sought to innovate care for people living with multiple long-term conditions [74], we aimed to capture and communicate the experience of care and use this to inspire service redesign. A tool was created to enable participants to share their experience through a bespoke visual map. The tool used the metaphor of a solar system with orbits representing each condition, and a range of symbols to represent the people (planets), tools (telescopes), and positive (shooting stars) and negative (black holes) aspects in their experiences of care. These categories were chosen based on previous research. The map was completed in a one-to-one session, where the DR captured the responses to the questions using the tool. Using the visual tool and through written annotation, the DR was able to co-interpret and produce the visual representation of the experience with the person.

\section{The Dissemination Space}

As in all good dissemination strategies, key messages need to be tailored to the target audience for knowledge translation "products" to be meaningful [43]. Within product development contexts, Visser et al. [80] have discussed the challenges of communicating the findings of PD research in engaging ways to support handover to a design team. Strategies include encouraging the design team to get involved in PD sessions, representing findings in the form of stories, and encouraging interaction with the insights by leaving some of the sense-making and conclusions to be made by the designers. We find these approaches also work to engage external audiences from non-design backgrounds.

Within the context of health and care, key audiences include the people who provide services including frontline practitioners and service managers, policy makers and strategic leaders, academics, and industry who seek to develop innovation. We need to create a range of outputs in different media to be relevant to the specific audience. The content of outputs varies depending on the audience, for example, across all projects we generate knowledge on both the health and care subject matter (research through design) and knowledge on design research methods and approaches (research into design). These types of knowledge have value for different communities across health, care, design and social sciences. Projects create a range of different outputs including website summary reports, downloadable project reports, lay summaries, visual outputs, videos, artefacts and engagement tools. Throughout our PD process, we translate insights into design opportunities, concepts and prototypes for different forms of innovation.

Illustration of the Dissemination Space: Within a project that explored the potential of person-owned data stores to support more integrated and person-centred care [76], the dissemination space aimed to engage the wider digital health and care community in discussing the potential of the concepts developed. Alongside four visual concepts for functionality supported by design rationale [58] from the perspectives of the people who receive and provide care, we created a digital prototype and roadmap for implementation. The prototype demonstrated the value of the technology in tackling information sharing challenges across services, and the roadmap distilled key questions for decision makers, in order to start a dialogue.

\section{DISCUSSION}

The Spaces model contributes to the landscape of PD organising principles and guidelines that currently fall short of considering how to support participation, or fail to consider participation beyond organising activities. Similar to Brandt [11], this model highlights the importance of not only the outcome of a design process but the value of the process itself. Focusing on the goal of participation, rather than a predetermined design outcome ensures that the process leaves room for emergence and change in direction based on the learning [8].

\section{A Model that Articulates Practice}

The Spaces model emerged as a means of articulating our PD process for health and care innovation. We struggled to represent our process as a linear, standardised set of activities. In drawing out the patterns across our projects, we saw that the underlying practice was in curating a sequence of bespoke engagements and creating the conditions to enable participants to meaningfully engage. In seeking to define these conditions we gave them a tangible and visual form: through reification we communicated the complex nature of our practice.

In describing the participatory mindset [68], and explaining the values that underpin $\mathrm{PD}$ we can communicate the nature of $\mathrm{PD}$ practice. However, there is a risk that the practice is seen as using 
tools and techniques "more or less at will" [12] which devalues the skill and sensitivity to context of the practitioner. The Spaces model is an attempt to articulate the tangible product of the practice, that of curating and designing the conditions for participation.

This ability to articulate practice is important when collaborating with other disciplines and integrating PD within multidisciplinary research projects. Social science researchers are increasingly being called upon to develop interventions as an outcome of research, and best practice suggests that interventions be designed in collaboration with the intended users [61]. We propose that the Spaces model can provide a scaffold for researchers who wish to employ a participatory or co-design element to their work, and can support design practitioners, DRs and students who are working in social innovation to instil the principles and value of $\mathrm{PD}$, and guide them in using these approaches.

\section{A Flexible Model that Scaffolds Participation}

In reviewing the different frameworks, models and lenses offered to scaffold participation, we found a prevalence towards linear, activity-focused frameworks, and lenses offering one perspective on participation. These are generally offered as starting points from which the DR can carefully select, adapt and appropriate to suit the context [13]. Despite this, there is a risk that these ways of scaffolding participation could limit the inherent creativity of DRs. As Brandt et al. [12] stress, when selecting the activities and tools for "the problem at hand", the DR needs to be ". . a aware of what is accomplished as particular tools and techniques become part of design practices".

Our positioning of the critical space near the top of the scaffold, indicates that this is often the pinnacle of what we are trying to achieve in meaningfully engaging participants. Participants need to understand the challenge and the opportunities identified, the possibilities of new technologies or ways of working, and relate these to their everyday lives or work. Assessing where participants are at currently against the goals of the critical space, and designing the process to scaffold this is essentially the practice of the DR and is where we feel the Spaces model makes an important contribution. However, the flexible, non-linear nature of the Spaces model allows for projects where participants can immediately engage with the critical space, and where power imbalances mean that the collaboration space is the real goal for meaningful participation and resolution of conflicting requirements. In this example, the analysis space could be employed as a mechanism to enable the collaboration space, using meaning making to achieve an understanding of other's points of view. Thus, the model ensures participation goes beyond inclusion and consultation to empowerment [48].

The model directs focus on the design of each space, inviting DRs to draw from the wealth of different methods and techniques to meet the aims and create the conditions for participation. This flexible structure avoids the need to separate the "intertwined...setting and solving the problem" into different phases [70] and can be adapted to suit the scale and duration of projects. The model provides a scaffold to integrate multiple perspectives by supporting interdisciplinarity and extends beyond existing definitions of 'space' held in HCI [60].
There are useful frameworks which give practical guidance about how to design participation $[67,80]$, but where we argue the Spaces model takes this further is in considering the more experiential and relational aspects of participation. By considering the 'space' as the focus of design, the model encourages creativity and attention to detail in designing the whole experience of participation, creating "interactive environments that scaffold the process" of participants contributing to the design [30].

\section{A Model that Supports Reflection}

Any PD process needs room for emergence and the use of reflection on progress to alter the plan for participation. We propose that the Spaces model offers a tool to reflect on progress, adapt and direct the aimed participation and outputs. As a tool for reflection at the end of the process, we suggest that the DR can consider how the design of each space achieved the goal, and what factors contributed to this. They can consider whether there may have been a quicker route to meet the project goal, and what parts of the process worked most effectively to engage. They can reflect on the combination, adaptation and extension of existing tools and techniques "to form the basis for yet newer PD practices" [12]. The model can also serve as a way to guide internal research, which may focus on the design of a particular space, or number of spaces.

\section{LIMITATIONS AND FURTHER RESEARCH}

A key limitation of the model is that it has not been evaluated for use as a tool to support the design and planning of a PD research process. Preliminary feedback suggests that the model has potential for use in providing a structure for research, however further testing is required. In addition, further research is required to explore more widely how this model could support reflection among designers, DRs, and the wider PD community and also to establish whether the model can also be used as a way to guide a PD process. Future work will explore the model with these communities in order to refine and develop a set of principles for deploying the Spaces model in practice.

\section{CONCLUSION}

This paper has introduced the Spaces for PD Innovation model, which resulted from a methodological reflection on PD research within the context of digital health and care. We have presented a critique of existing PD frameworks, models and lenses, and highlighted a gap in guidance that balances the need for a structured process, with the need for flexible and open models that support creative responses to the context. We explained the background and development of the model, and provided a detailed description, illustrated with project examples and discussed in relation to existing literature. Although further testing of the model is required, we propose that it offers an important contribution to the PD landscape to articulate practice, scaffold participation and enable reflective practice towards supporting meaningful participation. We suggest that this model provides scaffolding for the creative selection and adaptation of tools and techniques, with a holistic view of how they combine to achieve project and participation goals. Future research will engage with other DRs, practitioners, students and researchers from other disciplines to explore the implications for PD 
practice and teaching, and the integration of $\mathrm{PD}$ in multidisciplinary research collaborations.

\section{ACKNOWLEDGMENTS}

The Digital Health and Care Institute is part of the Scottish Funding Council's Innovation Centre Programme and is part funded by the Scottish Government. We gratefully acknowledge the design research team involved with the examples used to illustrate the model: Sanne Ree Barthels, Jeroen Blom, Sneha Raman, Angela Bruce, Cate Green, Tine Thorup and Jay Bradley.

\section{REFERENCES}

[1] Canan Akoglu, and Kathrina Dankl. 2019. Co-Creation for Empathy and Mutual Learning: A Framework for Design in Health and Social Care. CoDesign, 1-17.

[2] Paul Bate, and Glenn Robert. 2006. Experience-Based Design: From Redesigning the System around the Patient to Co-Designing Services with the Patient. Quality and Safety in Health Care 15 (5): 307-10.

[3] David Benyon. 2014. Spaces of Interaction, Places for Experience. Edited by John M Carroll. Synthesis Lectures on Human-Centered Informatics. Vol. 22. Morgan and Claypool.

[4] Erling Björgvinsson, Pelle Ehn, and Per Anders Hillgren. 2010. Participatory Design and 'Democratizing Innovation'. In Proceedings of the 11th Biennial Participatory Design Conference. ACM, Sydney, Australia, 41-50.

[5] Jeroen Blom, Tara French, and Gemma Teal. 2016. Interaction Space: Older Adults and in-Home Systems. In eTELEMED 2016, 24-28 Apr, Venice, Italy.

[6] Jeanette Blomberg, and Mark Burrell. 2002. An Ethnographic Approach to Design. In The Human-Computer Interaction Handbook: Fundamentals, Evolving Technologies, and Emerging Applications, edited by Andrew Sears and Julie A Jacko, Second, 965-88. Lawrence Erlbaum Associates.

[7] Mark Blythe. 2014. Research through Design Fiction: Narrative in Real and Imaginary Objects. In Proceedings of the SIGCHO Conference on Human Factors in Computing Systems. ACM, Toronto, ON, Canada, 703-712.

[8] Susanne Bødker, and Ole Sejer Iversen. 2002. Staging a Professional Participatory Design Practice: Moving PD beyond the Initial Fascination of User Involvement In Proceedings of the second Nordic conference on Human-computer interaction, NordiCHI. ACM, <monospace/>Århus, Denmark, 11-18.

[9] Simon Bowen. 2010. A Critical Artefact Methodology: Using Provocative Conceptual Designs to Foster Human-Centred Innovation. PhD Dissertation. Sheffield Hallam University.

[10] Simon Bowen, Kerry McSeveny, Eleanor Lockley, Daniel Wolstenholme, Mark Cobb, and Andy Dearden. 2013. How Was It for You? Experiences of Participatory Design in the UK Health Service. CoDesign, 9 (4): 230-46.

[11] Eva Brandt. 2006. Designing Exploratory Design Games: A Framework for Participation in Participatory Design? In Proceedings of the ninth Participatory Design Conference. ACM, Trento, Italy, 57-66.

[12] Eva Brandt, Thomas Binder, and Elizabeth B.N. Sanders. 2012. Tools and Techniques: Ways to Engage Telling, Making and Enacting. In Routledge International Handbook of Participatory Design, 165-201. Routledge.

[13] Tone Bratteteig, Keld Bødker, Yvonne Dittrich, Preben Holst Mogensen, and Jesper Simonsen. 2013. Methods: Organising Principles and General Guidelines for Participatory Design Projects. In Routledge International Handbook of Participatory Design, 117-44. Routledge, New York.

[14] Tim Brown. 2008. Design Thinking. Harvard Business Review 86 (6).

[15] Marion Buchenau, Marion, and Jane Fulton Suri. 2000. Experience Prototyping. In Proceedings of the Conference on Designing Interactive Systems: Processes, Practices, Methods, and Techniques, DIS. ACM, Brooklyn, New York, 424-33.

[16] Ana Maria Bustamante Duarte, Mehrnaz Ataei, Auriol Degbelo, Nina Brendel, and Christian Kray. 2019. Safe Spaces in Participatory Design with Young Forced Migrants. CoDesign, 1-23.

[17] Valerie Carr, Daniela Sangiorgi, Monika Büscher, Rachel Cooper, and Sabine Junginger. 2009. Clinicians as Service Designers? Reflections on Current Transformation in the UK Health Services. In First Nordic Conference on Service Design and Service Innovation, 31-42.

[18] John M Carroll. 1995. Scenario-Based Design: Envisioning Work and Technology in System Development. John Wiley \& Sons, Inc.

[19] George Chin, Mary Beth Rosson, and John M. Carroll. 1997. Participatory Analysis: Shared Development of Requirements from Scenarios. In Proceedings of the ACM SIGCHI Conference on Human factors in computing systems. ACM, Atlanta GA, 162-169.

[20] Campbell Christie. 2011. Commission on Future Delivery of Public Services.The Scottish Government.

[21] Chaloner Chute, and Tara French. 2019. Introducing Care 4.0: An integrated care paradigm built on 4.0 capabilities. International fournal of Environmental Research and Public Health (16) 12.

[22] Carla Cipolla and Roberto Bartholo. 2014. Empathy or inclusion: A dialogical approach to socially responsible design. International fournal of Design, 8(2), 87-100.

[23] Peter Coughlan, Jane Fulton Suri, and Katherine Canales. 2007. Prototypes as (Design) Tools for Behavioral and Organizational Change A Design-Based Approach to Help Organizations Change Work Behaviors. The fournal Of Applied Behavioral Science, Vol. 43 No. 1, March 2007 122-134.

[24] Yvonne Dittrich, and Olle Lindeberg. 2004. How Use-Oriented Development Can Take Place. Information and Software Technology 46 (9): 603-17.

[25] Sara Donetto, Paola Pierri, Vicki Tsianakas, and Glenn Robert. 2015. Experience Based Co-Design and Healthcare Improvement: Realizing Participatory Design in the Public Sector. Design fournal 18 (2): 227-48.

[26] Andrew Drain, Aruna Shekar, and Nigel Grigg. 2018. Insights, Solutions and Empowerment: A Framework for Evaluating Participatory Design. CoDesign, $1-21$.

[27] Allison Druin. 2002. The Role of Children in the Design of New Technology. Behaviour and Information Technology 21 (1): 1-25.

[28] Anthony Dunne. 2008. Hertzian Tales: Electronic Products, Aesthetic Experience, and Critical Design. PhD Thesis. Royal College of Art.

[29] Christiane Floyd, Fanny-Michaela Reisin, and Gerhard Schmidt. 1989. STEPS to Software Development with Users. In European Software Engineering Conference. Springer, Berlin, Heidelberg, 211-12.

[30] Christopher Frauenberger, Judith Good, and Wendy Keay-Bright. 2010. Phenomenology, a Framework for Participatory Design. In Proceedings of the 11th Biennial Participatory Design Conference. ACM, Sydney, Australia, 187-90.

[31] Christopher Frauenberger, Judith Good, Geraldine Fitzpatrick, and Ole Sejer Iversen. 2015. In Pursuit of Rigour and Accountability in Participatory Design. International fournal of Human Computer Studies 74: 93-106.

[32] Tara French, and Gemma Teal. 2015. Co-designing a digital directory of services. In The 5th International Conference on Current and Future Trends of Communication Technologies in Healthcare (ICTH), Berlin.

[33] Tara French, and Gemma Teal. 2015. Transforming healthcare through design-led innovation. In Proceedings of the Third European Conference on Design4Health 2015, Sheffield, 13-16 July 2013.

[34] Tara French, and Gemma Teal. 2016. Design for empathy within participatory design approaches. In Cumulus Hong Kong 2016: Open Design for E-very-thing,2124 November 2016, Hong Kong Design Institute.

[35] Tara French, Gemma Teal, and Sneha Raman. 2016. Experience Labs: Co-Creating Health and Care Innovations Using Design Tools and Artefacts. In 2016 Design Research Society 50th Anniversary Conference, 27 - 30 Jun 2016, Brighton, UK.

[36] Tara French, Gemma Teal, and Cara Broadley. 2017. The subtleties of care: illuminating relational care through design. In Does Design Care? An International Workshop of Design Thought and Action, 12-13 September 2017, Imagination, Lancaster University.

[37] William W. Gaver, John Bowers, Andrew Boucher, Hans Gellerson, Sarah Pennington, Albrecht Schmidt, Anthony Steed, Nicholas Villars, and Brendan Walker. 2004. The Drift Table: Designing for Ludic Engagement. In CHI'04 Extended Abstracts on Human Factors in Computing Systems. ACM, Vienna, 885-900.

[38] William W Gaver, Tony Dunne, and Elena Pacenti. 1999. Cultural Probes. Interactions 6 (February): 21-29.

[39] Victoria Gerrard, and Ricardo Sosa. 2014. Examining Participation. In Proceedings of the 13th Participatory Design Conference: Research Papers-Volume 1: 111-120. ACM.

[40] Elisa Giaccardi, and Gerhard Fischer. 2008. Creativity and Evolution: A Metadesign Perspective. Digital Creativity 19 (1): 19-32.

[41] Russell E. Glasgow, and Karen M. Emmons. 2007. How Can We Increase Translation of Research into Practice? Types of Evidence Needed. Annual Review of Public Health 28 (1): 413-33.

[42] Trisha Greenhalgh, Claire Jackson, Sara Shaw, and Tina Janamian. 2016. Achieving Research Impact Through Co-Creation in Community-Based Health Services: Literature Review and Case Study. Milbank Quarterly 94, no. 2 (2016): 392-429.

[43] Jeremy M. Grimshaw, Martin P. Eccles, John N. Lavis, Sophie J. Hill, and Janet E. Squires. 2012. Knowledge Translation of Research Findings. Implementation Science 7 (1).

[44] Kaj Grønbæk, Morten Kyng, and Preben Modensen. 1997. Toward a Cooperative Experimental System Development Approach. Computers and design in context, 201-238.

[45] Kursty Groves, and Oliver Marlow. 2016. Spaces for Innovation: The Design and Science of Inspiring Environments. Frame Publishers, Amsterdam.

[46] Christina N Harrington, and Anne Marie Piper. 2019. Deconstructing CommunityBased Collaborative Design: Towards More Equitable Participatory Design Engagements. In Proceedings of the ACM on Human-Computer Interaction, 3 (CSCW), 216.

[47] Karen Holtzblatt, and Hugh Beyer. 2015. Contextual Design: Evolved. Edited by John M Carroll. Synthesis Lectures on Human-Centred Informatics \#24. Morgan and Claypool. 
[48] Sofia, Hussain. 2010. Empowering Marginalised Children in Developing Countries through Participatory Design Processes. CoDesign 6 (2): 99-117.

[49] Alexandre Joyce, and Raymond L. Paquin. 2016. The Triple Layered Business Model Canvas: A Tool to Design More Sustainable Business Models. Fournal of Cleaner Production 135: 1474-86.

[50] Robert Jungk, and Norbert Müllert. 1987. Future Workshops: How to Create Desirable Futures. Inst. for Social Inventions.

[51] Finn Kensing, and Halskov Kim Madsen. 1991. Generating Visions: Future Workshops and Metaphorical Design. In Design at Work, edited by Morten Kyng and Joan Greenbaum.

[52] Finn Kensing, Jesper Simonsen, and Keld Bødker. 1998. Human - Computer Interaction MUST: A Method for Participatory Design MUST: A Method for Participatory Design. Human-Computer Interaction 13 (2): 167-98.

[53] Morten Kyng. 2010. Bridging the Gap Between Politics and Techniques: On the next Practices of Participatory Design. Scandinavian fournal of Information Systems 22 (221): 49-68.

[54] Dan Lockton, David Harrison, and Neville A Stanton. 2010. The Design with Intent Method: A Design Tool for Influencing User Behaviour. Applied Ergonomics 41 (3): 382-92.

[55] Louise Locock, Glenn Robert, Annette Boaz, Sonia Vougioukalou, Caroline Shuldham, Jonathan Fielden, Sue Ziebland, Melanie Gager, Ruth Tollyfield, and John Pearcey. 2014. Using a National Archive of Patient Experience Narratives to Promote Local Patient-Centred Quality Improvement: An Ethnographic Process Evaluation of 'accelerated' Experience-Based Co-Design. Fournal of Health Services Research \& Policy 19 (4): 200-207.

[56] Rachael Luck. 2018. What Is It That Makes Participation in Design Participatory Design? Design Studies 59: 1-8.

[57] Alastair S. Macdonald, Gemma Teal, and Paula J. Moynihan. 2010. A smarter food service for nutritionally vulnerable older adult hospital patients. In Proceedings of the 5th Cambridge Workshop on Universal Access and Assistive Technology, 169-177. Cambridge Engineering Design Centre.

[58] Allan MacLean, Richard M. Young, and Thomas P. Moran. 1989. Design Rationale: The Argument behind the Artifact. In ACM SIGCHI Bulletin Vol. 20, No. SI, 247-252. ACM.

[59] Andrew Monk, and Steve Howard. 1998. Methods \& Tools: The Rich Picture: A Tool for Reasoning about Work Context. Interactions 5 (2): 21-30.

[60] Michael Muller. 2007. Participatory Design: The Third Space in HCI. In The Human-Computer Interaction Handbook: 1087-1108. CRC Press.

[61] Nicola O'Brien, Ben Heaven, Gemma Teal, Elizabeth H. Evans, Claire Cleland, Suzanne Moffatt, Falko F. Sniehotta, Martin White, John C. Mathers, and Paula Moynihan. 2016. Integrating Evidence from Systematic Reviews, Qualitative Research, and Expert Knowledge Using Co-Design Techniques to Develop a WebBased Intervention for People in the Retirement Transition. Fournal of Medical Internet Research 18 (8).

[62] Antti Oulasvirta, Esko Kurvinen, and Tomi Kankainen. 2003. Understanding Contexts by Being There: Case Studies in Bodystorming. Personal and Ubiquitous Computing 7 (2): 125-34

[63] Sneha Raman, and Angela Tulloch. 2018. Ritual Respect: Co-designing care and emotional support around miscarriage.Project Report. Digital Health and Care Institute. Retrieved from www.futurehealthandwellbeing.org/ritualrespect
[64] Jim Rudd, Ken Stern, and Scott Isensee. 1996. Low vs. High Fidelity Prototyping Debate. Interactions 3 (1)

[65] Joanna Saad-Sulonen, Eva Eriksson, Kim Halskov, Helena Karasti, and John Vines. 2018. Unfolding Participation over Time: Temporal Lenses in Participatory Design. CoDesign 14 (1): 4-16.

[66] Elizabeth B. N. Sanders. 2000. Generative Tools for Co-Designing. In Collaborative Design, 3-12. Springer London.

[67] Elizabeth B. N. Sanders, Eva Brandt, and Thomas Binder. 2010. A Framework for Organizing the Tools and Techniques of Participatory Design. In Proceedings of the $11^{\text {th }}$ biennial Participatory Design Conference Series, 195-98. ACM.

[68] Elizabeth B. N. Sanders, and Pieter Jan Stappers. 2014. Probes, Toolkits and Prototypes: Three Approaches to Making in Codesigning. CoDesign 10(1), 5-14.

[69] Elizabeth B. N. Sanders, and Pieter Jan Stappers. 2008. Co-Creation and the New Landscapes of Design. CoDesign 4 (March): 1-16.

[70] Donald Schön. 1983. The Reflective Practitioner: How Professionals Think in Action. Edited by Basic Books. New York.

[71] Phoebe Sengers, Kirsten Boehner, Shay David, and Joseph Jofish Kaye. 2005. Reflective Design. In Proceedings of the 4th Decennial Conference on Critical Computing: Between Sense and Sensibility. ACM, Århus, Denmark, 49-58.

[72] Birger Sevaldson. 2008. Rich Design Research Space. FORMakademisk 1 (1): 28-44.

[73] Rachel Charlotte Smith, and Ole Sejer Iversen. 2018. Participatory Design for Sustainable Social Change. Design Studies 59: 9-36.

[74] Gemma Teal. 2018. A person-centred vision of care for people living with multiple long-term conditions. Project Report. Digital Health and Care Institute. Retrieved from www.futurehealthandwellbeing.org/modern-outpatients

[75] Gemma Teal, and Tara French. 2016. Designed Engagement. In2016 Design Research Society 50th Anniversary Conference, 27 - 30 Jun 2016, Brighton, UK

[76] Gemma Teal, Tara French, and Jay Bradley. 2018. Backpack.Project Report. Digital Health and Care Institute. Retrieved from www.futurehealthandwellbeing.org/backpack

[77] Gemma Teal, Tara French, Leigh Anne Heburn and Sneha Raman. 2016. Fostering Engagement Through Creative Collaboration. In Cumulus Open Design for Every-thing,21-24 Nov 2016, Hong Kong.

[78] Idong Usoro, Thomas Connolly, Sneha Raman, Tara French, and Stuart Caulfield. 2016. Using Games Based Learning to Support Young People With Learning Disabilities Stay Safe Online. In The 10th European Conference on Games Based Learning, 6-7 Oct 2016, University of the West of Scotland, UK.

[79] Christine Valenza, and Jan Adkins. 2009. Understanding Visual Thinking: The History and Future of Graphic Facilitation. Interactions 16 (4): 38-43.

[80] Froukje Sleeswijk Visser, Pieter Jan Stappers, Remko Vander Lugt, and Elizabeth B.N. Sanders. 2005. Contextmapping: Experiences from Practice. CoDesign, 1(2), 119-149.

[81] Dhaval Vyas, Dirk Heylen, and Anton Nijholt. 2009. Experiential Role of Artefacts in Cooperative Design. In Proceedings of the Fourth International Conference on Communities and Technologies. ACM, Pennsylvania, 105-14.

[82] Kristin L. Wiginton. 1999. Illness Representations: Mapping the Experience of Lupus. Health Education \& Behavior 26 (4): 443-53.

[83] Lucy Yardley, Leanne Morrison, Katherine Bradbury, and Ingrid Muller. 2015. The Person-Based Approach to Intervention Development: Application to Digital Health-Related Behavior Change Interventions. Fournal of Medical Internet Research 17 (1): e30. 